\title{
Mavenismo: un análisis de sus motivaciones
}

\author{
Beñat Urrutikoetxea Arrieta \\ Universidad de Granada \\ beurruti@ugr.es \\ https://orcid.org/0000-0003-0147-1501
}

\author{
Miguel Ángel Rodríguez Molina \\ Universidad de Granada \\ rmolina@ugr.es \\ http://orcid.org/0000-0002-3841-4706
}

\author{
José Alberto Castañeda García \\ Universidad de Granada \\ jalberto@ugr.es
}

http://orcid.org/0000-0003-0805-3628

\section{Mavenism: An analysis of their motivations}

\begin{abstract}
RESUMEN
En un mundo online sobrecargado, los usuarios buscan ayuda a la hora de tomar decisiones utilizando nuevas fuentes de información como referencia. Estas fuentes son identificadas como influenciadores $y$, entre ellos, los mavens son un tipo que tradicionalmente ha destacado en la literatura.

En este contexto, el uso de los influenciadores en las estrategias de marketing de las empresas se ha incrementado. Por lo tanto, el propósito de este estudio es conocer las motivaciones que determinan el comportamiento de los mavens.

Un total de 84 respuestas de influenciadores del sector del turismo fueron obtenidas analizando la influencia de las motivaciones en el comportamiento de los mavens a través del método de momentos generalizados. Los resultados muestran que los mavens tienen como motivaciones principales el altruismo, la empatía, la reciprocidad, la ganancia personal y la diversión. Las conclusiones obtenidas son valiosas a la hora de planificar estrategias de "Marketing con Influenciadores", ya que permite conocer mejor las motivaciones de este grupo clasificado como mavens. Al mismo tiempo, es de gran valor para aquellos profesionales que buscan influir a través de sus publicaciones en Internet.
\end{abstract}

PALABRAS CLAVE

Influenciadores, Mavenismo, Influencia, Medios sociales, Marketing digital, Marketing con influenciadores

\section{ABSTRACT}

In an overloaded online world, users seek help when making decisions using new sources of information as a reference. These sources are identified as influencers and among them mavens are a type that has traditionally stood out in the literature. In this context, the use of influencers in the marketing strategies of companies has increased. Therefore, the purpose of this study is to know the motivations that determine the behavior of the mavens. A total of 84 responses from influencers of the tourism sector were obtained by analyzing the influence of the motivations on the behavior of the mavens through the method of generalized moments. The results show that the main motivations of the mavens are altruism, empathy, the obligation to help others or reciprocity, personal gain and fun. The conclusions obtained are valuable to plan "Influencer Marketing" strategies, since it allows us to better understand the motivations of this group classified as mavens. At the same time, it is of great value for those professionals who seek to influence through their publications on the Internet.

\section{KEYWORDS}

Influencers, Mavens, Influence, Social media, Digital marketing, Influencer marketing net, (16), 51-60. D0I:10.31009/hipertext.net.2018.i16.09 


\section{Introducción}

Con la llegada de la Web 2.0 se ha generado una gran cantidad de contenido por parte de los usuarios (Sigala, 2008), lo que ha provocado que la Red se llene de información y el término sobrecarga de información, definido como la dificultad que una persona puede tener a la hora de tomar una decisión cuando tiene mucha información (Bontcheva, Gorrell y Wessels, 2013), se ha convertido en un concepto clave para entender el comportamiento de los usuarios en Internet (Benito-Rué, 2009).

Con la irrupción de la Web 2.0, el contenido generado por el usuario (UGC) y el boca-oído electrónico (eWOM) se ha modificado el modo en el que las personas buscan, encuentran, leen, recogen, comparten, desarrollan y consumen información (Aral, Dellarocas y Godes, 2013). Así, tanto en los modelos de propagación clásicos (Katz y Lazarsfeld, 1955), como en los nuevos modelos de medios sociales, hay un grupo de personas influyentes que actúan como intercambiadores de información fomentando la difusión de ideas (Burt, 1999). Estas personas influyentes se caracterizan por un elevado grado de persuasión (Bryman, 1993), un alto número de vínculos sociales (Goldenberg, Han, Lehmann y Hong, 2009) y un conocimiento superior sobre una materia (Lyons y Henderson, 2005). Merton (1957) descompone esta última característica en dos dimensiones: conocimiento en un área específica y conocimiento más general del mercado. Surgen así dos figuras: los líderes de opinión, que tienen un conocimiento amplio sobre algo específico, y los mavens, con un conocimiento más general referido al mercado (Feick y Price, 1987).

Tanto los líderes como los mavens pueden facilitar la comunicación de marketing de las empresas a través de un proceso con dos etapas (Bao y Chang, 2014): las empresas transmiten el mensaje a los influenciadores para que después éstos lo diseminen entre su amplia red de contactos. Esta estrategia resulta atractiva para las empresas debido a que la credibilidad de la información es más elevada, sobre todo si se compara con los medios tradicionales como la publicidad (Goldsmith, Clark y Goldsmith, 2006). De lo anterior se deriva que las empresas deberían estar interesadas en crear vínculos con los influenciadores para así alcanzar un mercado más amplio (Walsh, Gwinner y Swanson, 2004).

A pesar de ello, existen pocos trabajos cuyo objetivo haya estado centrado en estudiar los antecedentes que justifican el comportamiento de las personas influyentes, por lo que resulta necesario seguir profundizando en esta cuestión (Goldsmith et al., 2006). De manera particular, solamente dos trabajos han analizado el papel que juegan las motivaciones a la hora de compartir información por parte de los mavens (Walsh et al., 2004; Walsh y Mitchell, 2010). Sin embargo, como reconocen los propios autores, el número de motivaciones que se examina en estos trabajos seminales es muy limitado.
Considerando lo anterior, el objetivo de este trabajo es identificar las motivaciones que están relacionadas con el mavenismo ampliando el grado de conocimiento que se tiene sobre este perfil. Este trabajo es relevante para conocer mejor el funcionamiento de la influencia interpersonal en Internet obteniendo valiosos resultados tanto para las empresas que quieren planificar estrategias con influenciadores como para aquellos profesionales que buscan influir a través de sus publicaciones en la Red.

\section{Revisión de la literatura}

Los market mavens, o influyentes generales de mercado, son personas que tienen información acerca de muchos tipos de productos, lugares donde comprar y otras facetas del mercado, que inician conversaciones con los consumidores a la vez que responden a las solicitudes de información que los consumidores les hacen (Feick y Price, 1987). Mientras que los líderes de opinión son expertos en una categoría de producto (Lyons y Henderson, 2005), la influencia de los mavens se basa más en el conocimiento que tienen sobre el mercado en general (Brancaleone y Gountas, 2007).

En el caso de los medios online, este conocimiento se disemina según la predisposición de publicar contenido de cada usuario, siendo esta última una variable general que describe el comportamiento de los mavens. La principal conclusión que cabe destacar de la literatura revisada es que esta predisposición se debe a un conjunto variado de motivaciones que podrían explicar su comportamiento, no existiendo consenso en la literatura sobre su número y composición. De entre las propuestas, la de Syn y Oh (2015) se considera una de las más completas, pues recoge las motivaciones que han sido utilizadas en otros trabajos, llegando a proponer una decena de motivos que determinan la publicación de contenido online. En la tabla 1 tratamos de resumir las principales aportaciones respecto a los motivos para publicar activamente online por parte de cualquier individuo de Internet.

A pesar de la importancia de analizar las motivaciones que llevan a compartir contenido entre los mavens, los estudios llevados a cabo hasta el momento se han centrado principalmente en el estudio de sus características personales. Dos notables excepciones son los trabajos de Walsh et al. (2004) y Walsh y Mitchel (2010), donde se concluye que existen principalmente tres motivos que impulsan a los mavens: ayudar a los demás, obligación de compartir información y diversión. Sin embargo, como se pone de manifiesto en la Tabla 1 , las motivaciones para compartir información por parte de los individuos de Internet son más amplias, por lo que resulta conveniente completar el perfil de los mavens analizando otras motivaciones que sean relevantes para explicar su comportamiento. 


\begin{tabular}{|l|l|l|}
\hline Medio & Autores & Motivaciones \\
\hline \multirow{5}{*}{ Internet en general } & Daugherty, Eastin y Bright (2008) & Social, ego defensivo y expresiva de valor \\
\cline { 2 - 3 } & Hur, Kim, Karatepe y Lee (2017) & Estar informado, relaciones personales y entretenimiento \\
\cline { 2 - 3 } & Munar y Jakobsen (2014) & Altruismo, creación de comunidad \\
\cline { 2 - 3 } & Huang, Basu y Hsu (2010) & Obtener información, divulgar información y recordar lo vivido \\
\hline \multirow{3}{*}{ blog } & Wu y Pearce (2016) & $\begin{array}{l}\text { Altruismo, autorrealización, estatus personal, estatus social, diver- } \\
\text { Sión y autodocumentación }\end{array}$ \\
\hline \multirow{2}{*}{ Facebook y Twitter } & Syn y Lin (2008) & Diversión, estatus y altruismo \\
\cline { 2 - 3 } & Kang y Schuett (2013) & Diversión, internalización e identificación \\
\hline Youtube & Khan (2017) & $\begin{array}{l}\text { Diversión, eficacia, aprendizaje, estatus, empatía, beneficio personal, } \\
\text { altruismo, interés por la comunidad, compromiso social, reciprocidad }\end{array}$ \\
\hline
\end{tabular}

\section{Tabla 1. Motivaciones para publicar contenidos en medios online por parte de los usuarios en general. Elaboración propia.}

Los mavens se caracterizan por adquirir información del mercado y compartirla con los demás buscando la mejor opción disponible (Ruvio y Shoham, 2008). Para alcanzar este objetivo, seleccionan la información que les resulte más relevante (Geissler y Edison, 2005), lo que deja entrever una elevada autoeficacia. La autoeficacia ha sido definida como el juicio acerca de uno mismo para ejecutar una tarea con éxito (Bandura, 1997). En el contexto de las plataformas de redes sociales, podría argumentarse que los mavens comparten información para hacer visibles contenidos que creen que son valiosos. Según lo anterior, proponemos que:

H1. La autoeficacia está relacionada positivamente con la tendencia al mavenismo.

Otra de las razones por la que se comparte contenido en Internet está ligada al valor de la fama, esto es, el aumento de la popularidad (Rui y Whinston, 2012). La literatura ha puesto de manifiesto que esta motivación está relacionada con la figura de los bloggers, los nuevos líderes de opinión (Segev, Villar y Fiske, 2012; Sung y Kim, 2015), y no tanto al mavenismo, ya que a los mavens les importa más el nosotros que el yo, por lo que no lo tienen en cuenta en sus decisiones (Brancaleone y Gountas, 2007; Walsh et al., 2004). Por lo anterior, proponemos que:

H2. El estatus no está relacionado con la tendencia al mavenismo.

Por el contrario, los mavens encuentran en el deseo de ayudar a los demás una de sus principales motivaciones debido a su personalidad gregaria (Slama, Nataraajan y Williams, 1992; Walsh et al., 2004). Este comportamiento, ligado al altruismo (Ertekin y Atik, 2012), les lleva a proporcionar información sin esperar ninguna compensación a cambio (Oh, 2011). Tanto es así, que incluso Dichter (1966), uno de los primeros autores en analizar la figura de los mavens, los calificó como influenciadores de buena voluntad. Por lo tanto, planteamos que:
H3. El altruismo está relacionado positivamente con la tendencia al mavenismo.

Cuando los usuarios perciben que habrá algún tipo de beneficio, ya sea en forma de promoción de su trabajo (Baek, Holton, Harp y Yaschur, 2011) o recopilación de información interesante (Boyd, Golder, y Lotan, 2010), comparten más contenido online. En este entorno, cuando se ha analizado a los mavens, se ha encontrado que buscan la promoción (Alshawaf y Le, 2015) y el bienestar personal (Hwan, Leizerovici y Zhang, 2012). Lo anterior nos lleva a plantear que:

H4. El beneficio personal está relacionado positivamente con la tendencia al mavenismo.

Los mavens consumen gran cantidad de horas en Internet, lo que les garantiza relacionarse y aumentar su red de contactos (Yang, 2013), siendo la importancia del mantenimiento de esta red una razón más que les empuja publicar contenido (Laughlin y Macdonald, 2010). Por esta razón, planteamos que:

H5. El interés por la comunidad está relacionado positivamente con la tendencia al mavenismo.

Relacionado con la gestión de la red de contactos se encuentra la motivación de conocer gente nueva y estar en contacto con estas personas como otra de las razones para publicar contenidos (Syn y Oh, 2015). Los mavens se rigen por beneficios sociales (Price, Feick, y Higie, 1987) y tienen interés por actividades sociales siendo caracterizados como extrovertidos (Brancaleone y Gountas, 2007), por lo que proponemos que:

H6. El engagement social está relacionado positivamente con la tendencia al mavenismo.

Dos características sostienen la comunidad de los mavens. Por un lado, se encuentra la reciprocidad, definida como el ofrecimiento de ayuda con la expectativa de que el otro le devolverá el favor (Blau, 1964). Así, cuando un usuario proporciona información a otra persona, quien recibe la información 
se siente obligada a devolver el favor (Gatignon y Robertson, 1986). Esta motivación está relacionada con los mavens debido a su sentimiento de obligación de ayudar a los demás (Walsh et al., 2004). Por otro lado, está la habilidad de ponerse en el lugar de los demás. Esta característica surge cuando el usuario reconoce que puede coincidir en problemas con otros usuarios y sabe que necesitará ayuda en el momento que cambie la situación (Preece, 1999). Los mavens se relacionan con otros usuarios con los que comparten intereses (Hwan et al., 2012), siendo la homofilia una variable que describe su comportamiento. Por esto, y por su capacidad de abordar las necesidades de los demás (Walsh et al., 2004), planteamos que:

H7. La reciprocidad está relacionada positivamente con la tendencia al mavenismo.

H8. La empatía está relacionada positivamente con la tendencia al mavenismo.

Conocer las mejores prácticas y las últimas novedades se asocia con la participación en una comunidad (Wasko y Faraj, 2000). Por su condición de persona con experiencia sobre el mercado (Brancaleone y Gountas, 2007) y comportamiento exploratorio (Ruvio y Shoham, 2007), los mavens están en continuo aprendizaje (Geissler y Edison, 2005), por lo que se espera que ambas variables estén relacionadas positivamente:

H9. El aprendizaje está relacionado positivamente con la tendencia al mavenismo.

Finalmente, uno de los factores que más se atribuye al uso de las plataformas de redes sociales es la diversión (Lin y Lu, 2011), vista como una recompensa intrínseca a la hora de compartir contenido (Wasko y Faraj, 2000). Esta característica ya ha sido atribuida anteriormente a los mavens por parte de

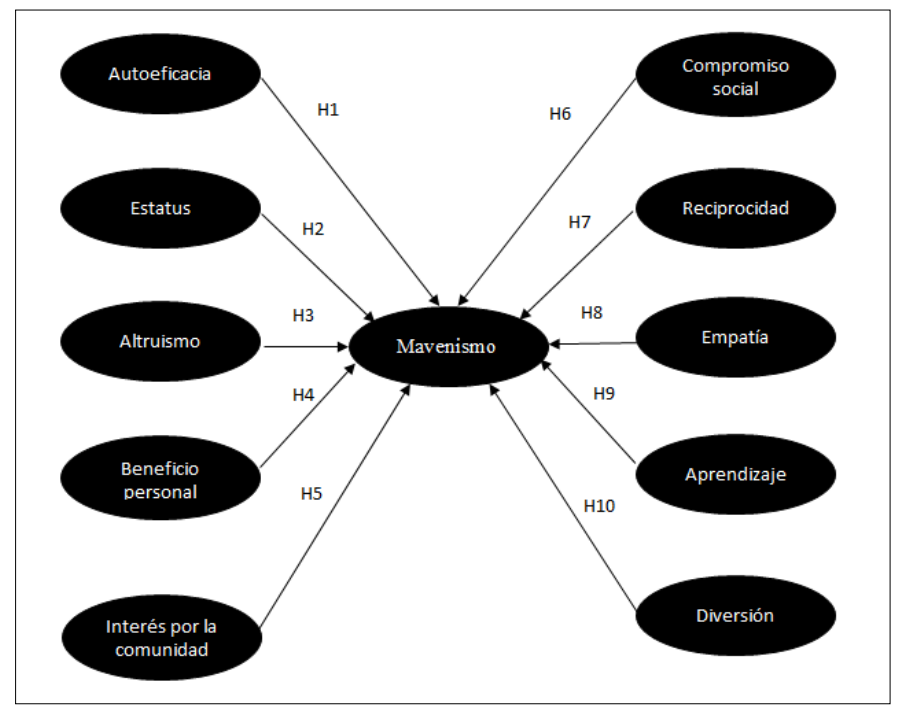

Figura 1. Modelo teórico del estudio. Elaboración propia. estudios previos (Walsh et al., 2004), por lo que esperamos que:

H10. La diversión está relacionada positivamente con la tendencia al mavenismo.

El modelo de la Figura 1 recoge de manera gráfica las hipótesis planteadas y se usará como marco de referencia para su posterior contraste.

\section{Metodología}

\subsection{Población y muestra}

La población de este trabajo se centra en personas influyentes que desarrollan su actividad en el sector turístico a través de una plataforma de microblogging como Twitter. Esta red social es la plataforma de microblogging más conocida en el mundo y se usa sobre todo por su valor informacional (Johnson y Yang, 2009), siendo idónea en el análisis del eWOM (Kim, Sung y Kang, 2014). Además, es la más usada en la literatura académica para identificar influenciadores en la Red (e.g. Bakshy, Hofman, Mason y Watts, 2011; Hung-Said y Arcila-Calderón, 2013).

Para la selección de la muestra se llevó a cabo una búsqueda de perfiles de personas influyentes a través de la herramienta Followerwonk incluyendo términos asociados al turismo como "viajes" "turismo" "travel" "travel blogger" "travel blog". Un total de 446 perfiles fueron obtenidos de este modo. Se envió a todos ellos la invitación para participar en este estudio, recibiendo 84 respuestas válidas, lo que supone una tasa de respuesta del 19\%. Las personas entrevistadas debían responder un cuestionario auto-administrado online que se elaboró usando el software Qualtrics. El trabajo de campo se realizó entre septiembre de 2016 y marzo de 2017.

\subsection{Instrumentos de medida}

El cuestionario empleado incluye principalmente dos grupos de variables: las motivaciones para compartir información y la tendencia al mavenismo.

Las motivaciones se han medido usando la propuesta de Syn y Oh (2015). Esta escala incluye diez motivaciones que justifican la predisposición general a compartir contenidos en medios sociales online y es una de las más completas hasta el momento. Cada una de estas motivaciones se encuentra medida con tres ítems usando una escala tipo Likert de siete posiciones.

Por su parte, la tendencia al mavenismo se ha medido a través de la escala propuesta por Feick y Price (1996) y consta de seis ítems medidos en una escala tipo Likert de siete posiciones. 
Aparte de lo anterior, se ha recogido información sobre el número de seguidores, puntuación Klout, número de tweets y horas diarias usando Twitter, lo que permitirá tener una idea más completa del perfil de la persona influyente que participa en este estudio.

\section{Resultados}

\subsection{Características de la muestra}

Los participantes del estudio son usuarios con una media de 7254 seguidores en Twitter y puntuación Klout superior a 40 puntos, un límite que algunos autores han propuesto para considerar a alguien como influenciador (Serrano-Puche, 2012). Además, son personas activas si se considera la infor-

\begin{tabular}{|c|c|c|}
\hline Variable & Media & D.T. \\
\hline Klout (de 0 a 100) & 56.10 & 10.16 \\
\hline Número de seguidores & 7254 & 17010.54 \\
\hline Número de Tweets & 13642 & 13568.54 \\
\hline Horas en Twitter & 1.56 & 1.27 \\
\hline
\end{tabular}

Tabla 2. Características de la muestra y utilización de Twitter. Elaboración propia.

mación publicada (13642 tweets de media) e invierten más de una hora y media al día en esta plataforma (Tabla 2).

\subsection{Estimación y evaluación del ajuste del modelo planteado}

Para contrastar las hipótesis planteadas, se ha estimado el modelo de la Figura 1 usando el análisis de componentes estructurados generalizados (GSCA) empleando 200 réplicas bootstrap. Los resultados muestran que la matriz de varianzas y covarianzas de la muestra y la reproducida por el modelo son muy similares (GFI=0.99; SRMR=0.09), por lo que se con- cluye que el ajuste del modelo es adecuado. La varianza de los indicadores y variables latentes explicada por el modelo (FIT) es igual a 0.58 , un valor relativamente elevado que indica que el modelo es aceptable.

Todas las variables latentes se han medido de manera reflectiva, aunque es preciso señalar que cuatro ítems de las motivaciones y uno de la escala de mavens fueron eliminados debido a su falta de fiabilidad. La evaluación del modelo de medida pone de manifiesto que todas las escalas poseen validez convergente y que existe validez discriminante entre las variables latentes del modelo estimado (Véase Anexo).

\subsection{Contraste de las hipótesis planteadas}

La tabla 3 muestra que los coeficientes entre el mavenismo, por un lado, y la autoeficacia, el altruismo, el beneficio personal, la reciprocidad, la empatía, el aprendizaje y la diversión , por otra parte, son estadísticamente significativos y su valor es moderado. De lo anterior se concluye que $\mathrm{H} 1, \mathrm{H} 2, \mathrm{H} 4, \mathrm{H} 7, \mathrm{H} 8$, H9 y 10 reciben apoyo empírico.

El estatus no está relacionado significativamente con el mavenismo . Este resultado es coherente con lo planteado en $\mathrm{H} 2$, no pudiendo rechazar dicha hipótesis.

En cambio, el interés por la comunidad y el compromiso social no están relacionados significativamente con el mavenismo , por lo que $\mathrm{H} 5$ y $\mathrm{H} 6$ deben ser rechazadas.

\section{Conclusiones}

Los resultados de este estudio demuestran que las motivaciones de los mavens son diversas a la hora de compartir información con los demás. Además de ayudar a los demás, sentirse obligados a compartir información y diversión (Walsh et al., 2004; Walsh y Mitchell, 2010), los mavens difunden información en los medios sociales online por otros motivos que incluyen la autoeficacia, el beneficio personal, la empatía y

\begin{tabular}{|l|c|c|c|c|c|c|}
\hline \multicolumn{1}{|c|}{ Relaciones propuestas } & Coeficiente & $\begin{array}{c}\text { Error } \\
\text { estándar } \\
\text { bootstrap }\end{array}$ & t-valor & $\begin{array}{c}\text { p-valor } \\
\text { (una cola) }\end{array}$ & Hipótesis & $\begin{array}{c}\text { ¿Se } \\
\text { rechaza? }\end{array}$ \\
\hline Autoeficacia > Mavenismo & 0.32 & 0.15 & 2.07 & 0.02 & 1 & No \\
\hline Estatus > Mavenismo & -0.06 & 0.14 & -0.42 & 0.66 & 2 & No \\
\hline Altruismo > Mavenismo & 0.25 & 0.11 & 2.27 & 0.01 & 3 & No \\
\hline Beneficio personal > Mavenismo & 0.23 & 0.13 & 1.80 & 0.04 & 4 & No \\
\hline Interés por la comunidad > Mavenismo & -0.06 & 0.10 & -0.62 & 0.73 & 5 & No \\
\hline Compromiso social > Mavenismo & 0.10 & 0.14 & 0.71 & 0.24 & 6 & Sí \\
\hline Reciprocidad > Mavenismo & 0.24 & 0.13 & 1.89 & 0.03 & 7 & Sí \\
\hline Empatía > Mavenismo & 0.29 & 0.10 & 2.77 & 0.00 & 8 & No \\
\hline Aprendizaje > Mavenismo & 0.24 & 0.12 & 1.99 & 0.02 & 9 & No \\
\hline Diversión > Mavenismo & 0.29 & 0.12 & 2.32 & 0.01 & 10 & No \\
\hline
\end{tabular}

Tabla 3. Coeficientes estimados (modelo estructural). Elaboración propia. 
el aprendizaje, coincidiendo con el planteamiento de las hipótesis del estudio. Sin embargo, los mavens no parecen difundir información sobre el mercado para mejorar su estatus, un mayor interés por la comunidad y un elevado compromiso social.

Una razón que justifica la ausencia de relación entre el interés por la comunidad, el compromiso social y el mavenismo en las plataformas de redes sociales, es que los mavens buscan un equilibrio entre el número de personas que forman su red de contactos y el grado de compromiso (Hwan et al., 2012). Sin embargo, este equilibrio es difícil de alcanzar en los medios sociales, y particularmente en Twitter, debido a que el número de contactos será elevado en la mayor parte de los casos. Además, este grado de compromiso se vincula con la fuerza de la relación (Granovetter, 1973), y en plataformas online dicho compromiso es, por lo general, mucho más débil en comparación con una comunidad del mundo offline (Chen, 2011; Bakshy, Rosenn, Marlow y Adamic, 2012).

Los mavens están motivados por el altruismo y el beneficio personal pero, en todo caso, este beneficio puede traducirse en aprendizaje y eficacia a la hora de afrontar las consultas que otros usuarios les hacen en su posición de influenciador, no como un modo de mejorar de estatus.

En definitiva, este trabajo contribuye a mejorar nuestro conocimiento sobre el perfil psicográfico, más que demográfico, que caracteriza a los mavens (Goldsmith et al., 2006), conociendo mejor su psicología y las motivaciones que subyacen a la influencia interpersonal.

Dada la importancia que han mostrado tener los influenciadores en el proceso de compra (IAB Spain, 2016), no es extraño que las empresas se hayan interesado en usarlos dentro de sus estrategias de marketing como posibles prescriptores (The Influence Marketer, 2017). En este sentido, las empresas podrían contribuir a favorecer algunas de las motivaciones relacionadas con los mavens para realizar campañas efectivas. Por un lado, en su relación con los influenciadores debería estar presente la diversión, ya que ésta es una condición necesaria de cualquier colaboración. Por otro lado, a pesar de su faceta altruista, el maven no trabaja sin un beneficio personal que puede traducirse por ejemplo en aprendizaje, algo que también puede estar presente cuando se diseña la campaña de marketing.

Finalmente, los mavens no están motivados para difundir información pensando en una mejora de estatus, una característica que es más propia del liderazgo de opinión donde la posición que ocupan dentro de la red social de la que forman parte es una cuestión importante para mantener su prestigio y su reputación. Los mavens, en cambio, comparten información de un modo más altruista y movidos por un beneficio personal basado en el aprendizaje.
Aunque este estudio tiene contribuciones importantes, también están presentes algunas limitaciones y genera algunas líneas de investigación interesantes para el futuro. La principal limitación está en la muestra, con 84 participantes heterogéneos en su comportamiento online (número de tweets, horas en Twitter) y en los indicadores de influencia (Klout o número de seguidores), pero que no son más que un reflejo de la realidad de este colectivo de influencers. Respecto a líneas futuras, además de la autopercepción de los influenciadores, para futuras investigaciones se debería añadir la percepción de los seguidores. De esta manera se comprenderá de manera más completa cómo se produce la influencia online. Finalmente, sería interesante incluir otros enfoques de influenciadores como los líderes de opinión (Hung-Said y Arcila-Calderón, 2013) o los hubs (López, Sicilia y Verlegh, 2017) para comprobar si existen diferencias en las motivaciones.

\section{Referencias}

Alshawaf, E., y Le, W. (2015). Understanding digital reputation on Instagram: A case study of social media mavens. En A. Mesquite, y P. Peres (Eds.), Proceedings of the 2nd European conference on social media ECSM 2015. Second European Conference on Social Media, Polytechnic Institute, (19-27). Porto, Portugal, 9-10 July.

Aral, S., Dellarocas, C., y Godes, D. (2013) Social media and business transformation: A framework for research, Information Systems Research, 24(1), 3-13.

Baek K., Holton A., Harp D., y Yaschur, C. (2011). The links that bind: Uncovering novel motivations for linking on Facebook. Computers and Human Behavior, (27), 2243-2248.

Bakshy, E., Hofman, J., Mason, W., y Watts, D. (2011). Everyone's an influencer: Quantifying influence on Twitter. Proceedings of the Fourth ACM International Conference on Web Search and Data Mining, Hong Kong, China, 9-12.

Bakshy, E., Rosenn, I., Marlow, C., y Adamic, L. (2012). The role of social networks in information diffusion. Proceedings of the 21st international conference on World Wide Web, 519-528.

Bandura, A. (1997). Self-efficacy: The exercise of Control. New York: Freeman.

Bao, T., y Chang, T. L. S. (2014). Finding disseminators via electronic word of mouth message for effective marketing communications. Decision Support Systems, (67), 21-29.

Benito-Rué, E. (2009). Infoxication 2.0. En THOMAS, M. (Ed.). Handbook of Research on Web 2.0 and Second Language Learning, (60-79). Pennsylvania: IGI-InfoSci.

Blau, P. (1986). Exchange and Power in Social Life. New York: Routledge.

Bontcheva, K., Gorrell, G., y Wessels,B. (2013). Social Media and Information Overload: Survey Results, Arxiv preprint arXiv: 1306.0813. Recuperado en https://arxiv.org/abs/1306.0813

Boyd, D., Golder, S., y Lotan, G. (2010). Tweet, tweet, retweet: Conversational aspects of retweeting on twitter. En System Sciences (HICSS), 43rd Hawaii International Conference, 1-10.

Brancaleone, V., y Gountas, J. (2007). Personality characteristics of market mavens. ACR North American Advances, (34), 522-527. 
Bryman, A. (1993). Charismatic leadership in business organizations: Some neglected issues. The Leadership Quarterly, 4 (3-4), 289-304.

Burt, R. S. (1999). The social capital of opinion leaders. Annals of the American Academy of Political and Social Science, 566, 37-54.

Chen, G. M. (2011). Tweet this: A uses and gratifications perspective on how active Twitter use gratifies a need to connect with others. Computers in Human Behavior, 27(2), 755-762.

Daugherty, T., Eastin, M-S., y Bright L. (2008). Exploring Consumer Motivations for Creating User-generated Content. Journal of Interactive Advertising, 8(2), 16-25.

Dichter, E. (1966). How word-of-mouth advertising works. Harvard Business Review, 44(6), 147-160.

Ertekin, Z.O., y Atik, D. (2012). Word-of-mouth communication in marketing: An exploratory study of motivations behind opinion leadership and opinion seeking. METU Studies in Development, 39(3) 323-345.

Feick, L. F., y Price, L. L. (1987). The market maven: A diffuser of marketplace information. Journal of Marketing, 51(1), 83-97.

Gatignon, H., y Robertson, T.S. (1986). An exchange theory model of interpersonal communication. Advances in Consumer Research, (13), 534-538.

Geissler, G. L., y Edison, S. W. (2005). Market mavens' attitudes towards general technology: Implications for marketing communications. Journal of Marketing Communications, 11(2), 73-94.

Goldenberg, J., Han, S., Lehmann, D. R., y Hong, J. W. (2009). The role of hubs in the adoption process. Journal of Marketing, 73(2), 1-13.

Goldsmith, R. E., Clark, R. A., y Goldsmith, E. B. (2006). Extending the psychological profile of market mavenism. Journal of Consumer Behaviour, 5(5), 411-419.

Granovetter, M. S. (1973). The strength of weak ties. American Journal of Sociology, 78(6), 1360-1380.

Hsu, C. L., y Lin, J. C. C. (2008). Acceptance of blog usage: The roles of technology acceptance, social influence and knowledge sharing motivation. Information, y Management, 45(1), 65-74.

Huang, Y., Basu C., y Hsu M.K. (2010). Exploring motivations of travel knowledge sharing on social network sites: An empirical investigation of U.S. college students. Journal of Hospitality Marketing y Management, 19(7), 717-734.

Hung-Said, E., y Arcila-Calderón, C. (2013). Online opinion leaders in Latin America and the Middle East: The case of the top 20 mostviewed twitter users. Informação \& Sociedade, 23(3), 143-157.

Hur, K., Kim, T., Karatepe, O. M., y Lee, G. (2017). An exploration of the factors influencing social media continuance usage and information sharing intentions among Korean travellers. Tourism Management, 63, 170-178.

Hwan, S., Leizerovici, G., y Zhang, S. (2012). Market mavens and networking: Benefits and costs of network participation. Advances in Consumer Research, (40), 593-594.

IAB Spain. (2016), Estudio ecommerce IAB Spain, España.

Johnson, P. R., y Yang, S. (2009). Uses and gratifications of Twitter: An examination of user motives and satisfaction of Twitter use. En Communication Technology Division of the annual convention of the Association for Education in Journalism and Mass Communication, Boston, MA. 1-32.
Kang, M., y Schuett, M. A. (2013). Determinants of sharing travel experiences in social media. Journal of Travel y Tourism Marketing, 30(1-2), 93-107.

Katz, E., y Lazarsfeld, P. F. (1955). Personal influence: The part played by people in the flow of mass communications. Piscataway, NJ: Transaction Publishers.

Khan, M. L. (2017). Social media engagement: What motivates user participation and consumption on YouTube? Computers in Human Behavior, (66), 236-247.

Kim, E., Sung, Y., y Kang, H. (2014). Brand followers' retweeting behavior on Twitter: How brand relationships influence brand electronic word-of-mouth. Computers in Human Behavior, (37), 18-25.

Laughlin, J. D., y MacDonald, J. B. (2010). Identifying market mavens online by their social behaviors in community-generated media. Academy of Marketing Studies Journal, 14(1), 55-70.

Lin, K., y Lu H. (2011). Why people use social networking sites: An empirical study integrating network externalities and motivation theory. Computers in Human Behavior, (27), 1152-1161.

López, M., Sicilia, M., y Verlegh, W.J. (2017). Click like if you like it": The effect of directional posts on social network sites. Online Information Review, 41(5), 672-690.

Lyons, B., y Henderson, K. (2005). Opinion leadership in a computer-mediated environment. Journal of Consumer Behaviour, 4(5), 319-329.

Merton, R.K. (1957). Social Theory and Social Structure (Revised edition). Glencoe, IL: Free Press.

Munar, A.M., y Jacobsen J.K.S. (2014). Motivations for sharing tourism experiences through social media. Tourism Management, (43), 46-54.

Oh, S. (2012). The characteristics and motivations of health answerers for sharing information, knowledge, and experiences in online environments. Journal of the Association for Information Science and Technology, 63(3), 543-557.

Preece, J. (2000). Online communities: Designing usability, supporting sociability. Chichester, UK: John Wiley.

Price, L. L., Feick, L. F., y Higie, R. A. (1987). Information sensitive consumers and market information. Journal of Consumer Affairs, 21(2), 328-341.

Rui, H., y Whinston, A. (2012). Information or attention? An empirical study of user contribution on Twitter. Information Systems and e-Business Management, (10), 309-324.

Ruvio, A., y Shoham, A. (2007). Innovativeness, Exploratory Behavior, Market Mavenship, and Opinion Leadership: An Empirical Investigation in the Asian Context. Psychology y Marketing, 24(8), 703-729.

Segev, S., Villar, M. E., y Fiske, R. (2012). Understanding opinion leadership and motivations to blog: Implications for public relations practice. Public Relations Journal, 6(5), 1-31.

Serrano-Puche, J. (2012). Herramientas web para la medición de la influencia digital: análisis de Klout y PeerIndex. El Profesional de la Información, 21(3), 298-303.

Sigala, M. (2008). Web 2.0, social marketing strategies and distribution channels for city destinations: Enhancing the participatory role of travellers and exploiting their collective intelligence. En M. Gascó-Hernández, T. Torres-Coronas (Eds.), Information communication technologies and city marketing: Digital opportunities for cities around the world. IDEA Publishing. 
Slama, M., Nataraajan, R., y Williams, T. G. (2015). Market mavens and the relationship between smart buying and information provision: An exploratory study. En Proceedings of the 1992 Academy of Marketing Science (AMS) Annual Conference, 90-93.

Sung, H., y Kim, E. Y. (2015). A Comparative Analysis of Psychological Factors for Predicting Market Mavenism and Fashion Leadership. Fashion Business, 19(5), 77-92.

Syn, S.Y., y Oh, S. (2015). Why do social network site users share information on Facebook and Twitter? Journal of Information Science, 41 (5), 553-569.

The Influence Marketer (2017). 150+ Influencer Marketing Agencies, Platforms, Tools, Companies. Recuperado de http://theinfluencemarketer.com/133-influencer-marketing-agencies-platforms-tools-companies

Walsh, G., Gwinner, K., y Swanson, S. (2004). What makes mavens tick? Exploring the motives of market mavens' initiation of information diffusion. Journal of Consumer Marketing, 21(2), 109-122.

Walsh, G., y V.W. Mitchell (2010). Identifying, segmenting and profiling online communicators in an internet music context. International Journal of Internet Marketing and Advertising, 6(1), 41-64.

Wasko, M.M., y Faraj, S. (2000). It is what one does: Why people participate and help others in electronic communities of practice. Journal of Strategic Information Systems, (9), 155-173.

Wu, M.Y., y Pearce, P.L. (2016). Tourism blogging motivations: Why do Chinese tourists create little "Lonely Planets"? Journal of Travel Research, 55(4), 537-549.

Yang, H. (2013). Market mavens in social media: Examining young Chinese consumers' viral marketing attitude, eWOM motive, and behavior. Journal of Asia-Pacific Business, 14(2), 154-178.

\section{NOTA}

Esta investigación ha sido posible gracias al apoyo del proyecto: "El efecto de las recomendaciones en el comportamiento del turista y en el valor de marca del destino. I+D+I ECO2015-65306-R" (MINECO/FEDER)

\section{CV}

Beñat Urrutikoetxea Arrieta. Es estudiante de Doctorado de Ciencias Económicas y Empresariales de la Universidad de Granada donde analiza el comportamiento del consumidor en el mundo online centrándose en el terreno de los medios sociales: los blogs y plataformas de redes sociales. En la temática de los blogs ha presentado un trabajo en el 19th World Marketing Congress de Academy of Marketing Science en París al mismo tiempo que tiene un artículo en revisión en una revista de impacto. Mientras que las plataformas redes sociales han sido el foco de su tesis. Además ha formado parte de la organización de las II Jornadas de Jóvenes Investigadores: Fomentando la Interdisciplinariedad (JIFFI) y es uno de los coordinadores de la tercera edición convertido en congreso nacional que se celebrará el 20, 21 y 22 de junio de 2018. Actualmente trabaja como técnico de emprendimiento en la Coordinación General de Emprendimiento de la Universidad de
Granada.

Miguel A. Rodríguez Molina. Es Profesor Titular de la Universidad de Granada. Su investigación se ha centrado sobre el comportamiento del consumidor, el turismo y las nuevas tecnologías de la información y la comunicación. Sus trabajos han sido publicados en revistas como Tourism Management, Journal of Travel Research, International Journal of Hospitality and Tourism Management, Current Issues in Tourism, The Service Industries Journal, Internet Research, Cities, Online Information Review, Journal of Advertising Research, Journal of Small Business Management, Journal of Sustainable Tourism o International Journal of Uncertainty, Fuzziness and Knowledge-Based Systems. Actualmente su interés se centra en conocer los factores que motivan el liderazgo de opinión en redes sociales y su relación con los seguidores, así como la eficacia que tienen los sistemas de recomendación personales en comparación con los sistemas de recomendación impersonales.

José Alberto Castañeda García. Es Profesor Titular en la Universidad de Granada. El tópico de investigación principal durante los últimos años ha sido el análisis del comportamiento del individuo en el mercado electrónico con especial enfoque, aunque no único, en el sector del turismo. Es autor de artículos en las principales revistas de turismo como Tourism Management, Journal of Travel Research o Current Issues in Tourism, y en revistas más específicas relacionadas con las tecnologías de la información como Information y Management, Internet Research u Online Information Review. En los últimos años ha coordinado, junto con Miguel A. Rodríguez, varios proyectos de investigación en la línea de investigación Internet y Turismo. 


\section{Anexos}

\begin{tabular}{|c|c|c|c|c|c|c|c|}
\hline Constructo & Variables/ítems & Coef. & SE & $\begin{array}{l}\text { t-va- } \\
\text { lor }\end{array}$ & $\begin{array}{l}\mathrm{p}-\mathrm{va}- \\
\text { lor }\end{array}$ & $\begin{array}{l}\text { Fiabi- } \\
\text { lidad }\end{array}$ & AVE \\
\hline \multirow{2}{*}{ Diversión } & Es divertido & 0.93 & 0.02 & 48.95 & 0.00 & \multirow{2}{*}{0.92} & \multirow{2}{*}{0.85} \\
\hline & Me hace sentirme feliz & 0.91 & 0.04 & 24.62 & 0.00 & & \\
\hline \multirow{3}{*}{ Autoeficacia } & $\begin{array}{l}\text { Me siento productivo creando y buscando } \\
\text { información interesante para otros }\end{array}$ & 0.94 & 0.02 & 55.53 & 0.00 & \multirow{3}{*}{0.94} & \multirow{3}{*}{0.84} \\
\hline & Me siento útil distribuyendo información interesante a otros & 0.93 & 0.02 & 54.59 & 0.00 & & \\
\hline & Es una forma de auto realización personal & 0.88 & 0.03 & 32.41 & 0.00 & & \\
\hline \multirow{3}{*}{ Aprendizaje } & Aprendo sobre temas en los que estoy interesado & 0.85 & 0.05 & 18.91 & 0.00 & \multirow{3}{*}{0.93} & \multirow{3}{*}{0.82} \\
\hline & $\begin{array}{l}\text { Me ayuda a saber la información en la } \\
\text { que la gente está interesada }\end{array}$ & 0.94 & 0.02 & 42.64 & 0.00 & & \\
\hline & Estoy al tanto de información actualizada & 0.92 & 0.02 & 57.44 & 0.00 & & \\
\hline \multirow{3}{*}{$\begin{array}{l}\text { Beneficio } \\
\text { personal }\end{array}$} & Me ayuda a promocionar un producto/marca/proyecto & 0.82 & 0.04 & 20.58 & 0.00 & \multirow{3}{*}{0.88} & \multirow{3}{*}{0.71} \\
\hline & Me ayuda a vender un producto o servicio & 0.88 & 0.04 & 22.62 & 0.00 & & \\
\hline & Es parte de mi trabajo & 0.82 & 0.06 & 13.27 & 0.00 & & \\
\hline \multirow{3}{*}{ Altruismo } & $\begin{array}{l}\text { Quiero ayudar a otros a que encuentren la } \\
\text { información que están buscando }\end{array}$ & 0.84 & 0.04 & 22.18 & 0.00 & \multirow{3}{*}{0.91} & \multirow{3}{*}{0.77} \\
\hline & $\begin{array}{l}\text { La gente debería ayudarse mutuamente } \\
\text { en la búsqueda de información }\end{array}$ & 0.91 & 0.03 & 35.12 & 0.00 & & \\
\hline & Me gusta ayudar a los demás & 0.88 & 0.02 & 36.79 & 0.00 & & \\
\hline \multirow{2}{*}{ Empatía } & $\begin{array}{l}\text { Empatizo con aquellos que tienen proble- } \\
\text { mas a la hora de encontrar información }\end{array}$ & 0.88 & 0.04 & 19.89 & 0.00 & \multirow{2}{*}{0.88} & \multirow{2}{*}{0.79} \\
\hline & $\begin{array}{l}\text { La gente encuentra buena informa- } \\
\text { ción si yo comparto mis publicaciones }\end{array}$ & 0.90 & 0.04 & 25.77 & 0.00 & & \\
\hline \multirow{2}{*}{$\begin{array}{l}\text { Interés por la } \\
\text { comunidad }\end{array}$} & Ayuda a promover los temas en los que estoy interesado & 0.89 & 0.03 & 34.08 & 0.00 & \multirow{2}{*}{0.88} & \multirow{2}{*}{0.79} \\
\hline & Contribuye al campo en el que soy experto & 0.89 & 0.02 & 37.17 & 0.00 & & \\
\hline \multirow{3}{*}{$\begin{array}{l}\text { Compromiso } \\
\text { social }\end{array}$} & Me ayuda a comunicarme con otros & 0.93 & 0.03 & 35.73 & 0.00 & \multirow{3}{*}{0.93} & \multirow{3}{*}{0.81} \\
\hline & Me ayuda a colaborar con otros en la comunidad de Twitter & 0.87 & 0.05 & 17.48 & 0.00 & & \\
\hline & Me siento identificado con otros & 0.90 & 0.04 & 23.68 & 0.00 & & \\
\hline \multirow{3}{*}{ Estatus } & Me agrada ver que el número de seguidores crece & 0.81 & 0.07 & 11.63 & 0.00 & \multirow{3}{*}{0.89} & \multirow{3}{*}{0.73} \\
\hline & Me gustaría ser reconocido como un contribuidor al turismo & 0.88 & 0.03 & 26.58 & 0.00 & & \\
\hline & Mi reputación aumenta través de mis publicaciones & 0.86 & 0.05 & 15.96 & 0.00 & & \\
\hline \multirow{2}{*}{ Reciprocidad } & $\begin{array}{l}\text { Quiero devolver el favor, ya que suelo encontrar buena } \\
\text { información en las publicaciones de los demás }\end{array}$ & 0.92 & 0.02 & 45.75 & 0.00 & \multirow{2}{*}{0.92} & 85 \\
\hline & $\begin{array}{l}\text { Creo que puedo conseguir información intere- } \\
\text { sante en las publicaciones de los demás }\end{array}$ & 0.93 & 0.02 & 44.10 & 0.00 & & J \\
\hline & $\begin{array}{l}\text { Me gusta hablar de nuevos produc- } \\
\text { tos y marcas entre mis seguidores }\end{array}$ & 0.75 & 0.07 & 11.15 & 0.00 & & \\
\hline & $\begin{array}{l}\text { Me gusta ayudar a los demás facilitándoles infor- } \\
\text { mación acerca de muchos tipos de productos }\end{array}$ & 0.83 & 0.05 & 16.54 & 0.00 & & \\
\hline Mavenismo & $\begin{array}{l}\text { La gente me pide información acerca de alter- } \\
\text { nativas para la compra de productos }\end{array}$ & 0.87 & 0.02 & 37.74 & 0.00 & 0.91 & 0.67 \\
\hline & $\begin{array}{l}\text { Si alguien me pregunta donde realizar la mejor compra a } \\
\text { partir de una gran variedad de productos, podría aconsejarle }\end{array}$ & 0.77 & 0.06 & 12.39 & 0.00 & & \\
\hline & $\begin{array}{l}\text { Mis seguidores creen que soy una buena fuente de infor- } \\
\text { mación cuando salen nuevos productos o marcas }\end{array}$ & 0.88 & 0.03 & 29.27 & 0.00 & & \\
\hline
\end{tabular}

Tabla 4. Validez convergente de las escalas (fiabilidad individual, fiabilidad compuesta y varianza extraída). Elaboración propia. 


\begin{tabular}{|c|c|c|c|c|c|c|c|c|c|c|c|}
\hline & (1) & (2) & (3) & (4) & (5) & (6) & (7) & (8) & (9) & (10) & (11) \\
\hline Diversión (1) & 0.85 & & & & & & & & & & \\
\hline Autoeficacia (2) & 0.28 & 0.84 & & & & & & & & & \\
\hline Aprendizaje (3) & 0.17 & 0.41 & 0.82 & & & & & & & & \\
\hline Beneficio personal (4) & 0.00 & 0.01 & 0.08 & 0.71 & & & & & & & \\
\hline Altruismo (5) & 0.09 & 0.24 & 0.30 & 0.04 & 0.77 & & & & & & \\
\hline Empatía (6) & 0.09 & 0.07 & 0.10 & 0.02 & 0.17 & 0.79 & & & & & \\
\hline Interés por la comunidad (7) & 0.00 & 0.04 & 0.08 & 0.07 & 0.12 & 0.23 & 0.79 & & & & \\
\hline Compromiso social (8) & 0.16 & 0.18 & 0.24 & 0.04 & 0.19 & 0.14 & 0.24 & 0.81 & & & \\
\hline Estatus (9) & 0.04 & 0.14 & 0.05 & 0.07 & 0.09 & 0.24 & 0.25 & 0.20 & 0.73 & & \\
\hline Reciprocidad (10) & 0.02 & 0.09 & 0.04 & 0.05 & 0.16 & 0.10 & 0.14 & 0.09 & 0.29 & 0.85 & \\
\hline Tendencia al mavenismo (11) & 0.09 & 0.00 & 0.06 & 0.09 & 0.01 & 0.12 & 0.03 & 0.07 & 0.05 & 0.06 & 0.67 \\
\hline
\end{tabular}

Tabla 5. Validez discriminante de las escalas (AVE en la diagonal principal y correlaciones al cuadrado en la diagonal inferior). Elaboración propia.

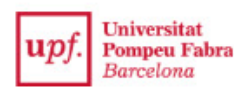
Barcelona

\begin{tabular}{|l|l|}
\hline Overview & $\boldsymbol{\oplus}$ \\
\hline Academic information & $\boldsymbol{\Theta}$ \\
\hline Apply & $\boldsymbol{\Theta}$ \\
\hline Tuition fees & $\boldsymbol{\Theta}$ \\
\hline Enrol & $\boldsymbol{\Theta}$ \\
\hline Timetable 2017-2018 & $\boldsymbol{\oplus}$ \\
\hline Scolarships and grants & $\boldsymbol{\oplus}$ \\
\hline
\end{tabular}

master.dcom@upf.edu
Master's Degree in Social Communication

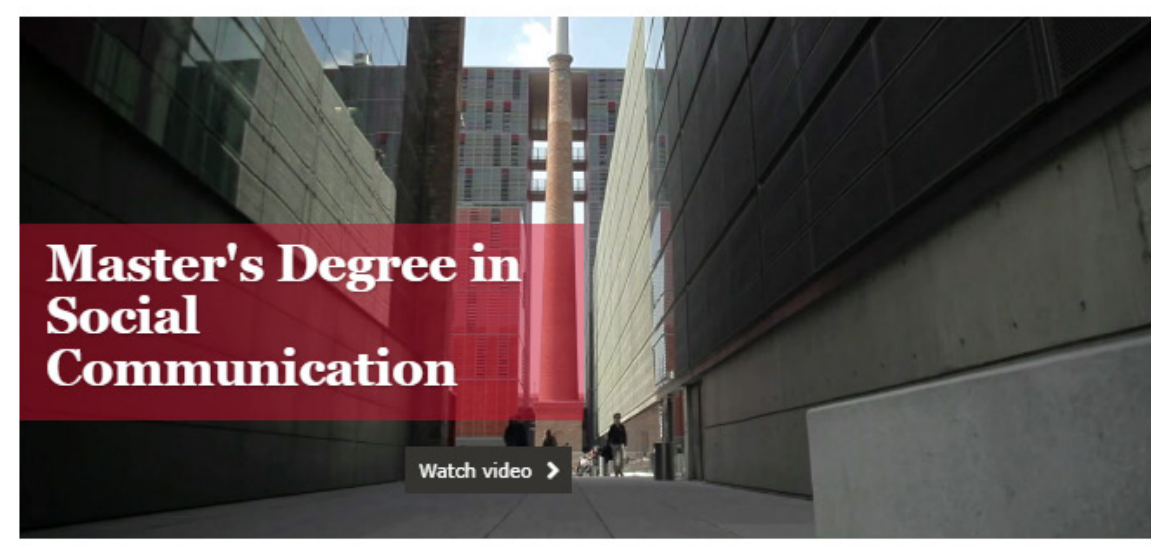

